\title{
Research of Wireless Network Performance for Digital Manufacturing
}

\author{
Yuntao Duan ${ }^{1}$, Siping $\mathrm{Hu}^{2}$, Shejie $\mathrm{Lu}^{*}, 2$ \\ ${ }^{1}$ Nanyang Institute of Technology, Nanyang, 473000, China \\ ${ }^{2}$ Hubei University of Science and Technology, Xianning, 430070, China
}

Keywords: WLAN, digital manufacturing, throughput, performance optimization

\begin{abstract}
In the IEEE 802.11 WLAN, as the number of nodes increases, the collision will increase accordingly, the channel utilization will decrease, and the total throughput will not increase and decrease, resulting in system performance degradation. This paper designs a simple and effective scheme based on the root cause of the problem - the dynamic time slot algorithm based on the number of nodes (DSTBNN). Then, through in-depth theoretical analysis, it is proved that the method can effectively improve system throughput and improve network performance. Finally, using NS2 simulation software, several simulation experiments were carried out in various scenarios. After analyzing the experimental results, the algorithm was proved to be simple and effective. The network system performance can be optimized according to the number of nodes, the system throughput is improved, and the network performance is significantly improved.
\end{abstract}

\section{Introduction}

Wireless networks for the digital manufacturing industry not only have great potential for energy savings, but also create opportunities for the high degree of flexibility and cost advantages of industrial system installation and operation. It is expected that by 2016, installation costs will be reduced by $80 \%$ and operating costs will be reduced by $50 \%$. As an access technology for digital manufacturing, wireless network has the following advantages compared with wired network technology: (1) Low cost: no cable connection is required, and the cable cost is saved by about 30 100 USD/m. During the installation and maintenance process, the trouble of installing and replacing the cable is avoided. Compared with the wired method, the wireless deployment, whether it is the physical cost of the signal transmission medium or the manpower and time cost in the deployment process, is much cheaper. (2) High flexibility and easy deployment: Because wireless networks adopt wireless transmission and reception technology, they completely get rid of the constraints of cables. Therefore, wireless networks have high flexibility and are fast, flexible and convenient according to the different needs of different users. Reorganize wireless network systems with low cost, making them easy to apply to new manufacturing environments. (3) High reliability and easy maintenance: cables have low reliability and high maintenance complexity. The use of wireless technology will prevent such system failures and improve the reliability of system operation. (4) Practicality: The low-power wireless communication module and wireless communication protocol can make the lifetime of the wireless network last for a long time, ensuring the practicality of the wireless network. (5) Wide coverage of use: There are some monitoring and data collection requirements in actual manufacturing, and the traditional wired method will not be able to connect to the target, or even if it is connected to the target, the maintenance will be quite difficult. For example, monitoring of rotating parts, large tanks or where hundreds or thousands of monitoring points are required. In these cases, if wireless networks are used, data that cannot be measured by traditional wired methods can also be collected by the monitoring network.

Therefore, wireless network technology is well suited as a perception and access to digital manufacturing systems. This paper focuses on the research and optimization of the problems of wireless access technology for digital manufacturing, and provides necessary and reliable technical support for manufacturing resource sharing, transaction and collaboration based on digital manufacturing systems. 
Digital manufacturing fully integrates the latest research results and advanced manufacturing models in the information field, and encapsulates relevant manufacturing resources in the manufacturing life cycle into digital resources. The perception and access of manufacturing equipment resources is the key to the virtualization of digital manufacturing physical resources. The security and reliability of manufacturing equipment resource awareness and access are important guarantees for the convergence, sharing and optimal configuration of manufacturing resources.

\section{Related research}

Literature [2] found through research that when the number of nodes changes within a certain range, the same optimized protocol parameters can be used, and the performance of the network can be nearly optimal. Based on this finding, the literature [2] proposes to use the interval measurement and estimation to replace the accurate measurement of system parameters in other algorithms. A DOOR (dynamic optimization on range) based on state detection and node number interval is designed. For the first time, it is proposed to replace the accurate measurement of network parameters in the previous algorithm based on the measurement and estimation of the interval. According to the analysis of the relevant model, the number of nodes is divided into multiple intervals, and the optimized network parameters of the intervals are calculated respectively. When the number of nodes does not change much and does not exceed the original interval, there is no need to adjust the relevant network parameters. Only when the number of nodes exceeds the original interval, the network parameters are dynamically adjusted according to the interval in which they are located, thereby achieving the purpose of improving network performance. The algorithm is simple, has low overhead, and is suitable for practical and complex wireless network environments. Compared with IEEE 802.11, the delay and throughput of the algorithm are significantly improved. The advantages of the algorithm are as follows: 1) It can reduce the dependence of the algorithm on the accurate measurement of network parameters; 2) When the number of nodes falls within a certain interval, the relevant parameters can be re-estimated and replaced. Saves on overhead.

According to the two-dimensional Markov model proposed by G Bianchi, the literature [3] deeply analyzes the performance of the frame length under the error channel to the saturation throughput of IEEE 802.11 DCF. The literature [3] pointed out that because the IEEE 802.11 DCF protocol has a relatively large overhead when the data frame is short, the utilization of the wireless channel is reduced. In addition, under the error channel, if the data frame is long, the probability of error of the data frame during transmission increases. Therefore, under a certain bit error rate, the optimal data frame length can maximize the throughput of the network. By deeply studying the relationship between the number of stages and the relationship between BER and optimal frame length, a simple transmission method based on network load conditions for frame length control is proposed. The control frame length is divided according to the BER interval, and the modification to the IEEE 802.11 protocol is small. At the same time, this strategy can increase the total throughput of the system.

Literature [4] proposed a MAC protocol based on collision avoidance. This method is based on the idea of polling [64]. The current sending node specifies the next sending node according to a certain strategy, so that collision can be avoided. The next node MAC layer address is added in the RTS and CTS, respectively. Moreover, each node needs to maintain a table of active nodes, which include active nodes and their transmission rates. The node currently transmitting data selects the next node from its active user list that needs to send data according to a certain policy. The policy of selecting the next node is described as follows. In order to ensure the fairness of time, the selection is based on the rate ratio in the system. Suppose $\mathrm{N}[\mathrm{j}]$ is the jth node in the network, its transmission rate is $R[j]$, the sum of the transmission rates of all nodes is sum $(R[j])$, and the node $N[j]$ is taken as $R[j] /$ sum (The probability of $R[j]$ ) is chosen as the next node. In transmission mode, the selected node begins transmitting data after the PIFS interval. In the competition mode, the interference mechanism is used, and the selected node initiates transmission after the DIFS time interval and backoff. Through this strategy, the channel collision probability is effectively reduced, and the 
purpose of improving network throughput is achieved. However, the disadvantage of this method is that each node needs to maintain a list of active nodes instead of being coordinated by the AP, which increases the burden and redundancy of the network and wastes valuable wireless network resources.

\section{Dynamic slot time algorithm based on number of nodes (DSTBNN)}

In order to be compatible with the existing standards, the dynamic slot algorithm based on the number of nodes changes the DCF mechanism of IEEE 802.11 to a small extent. The main difference is that when the number of nodes in the network is changed, the network parameters are dynamically adjusted according to the number of nodes. Achieve improved network performance.

The workflow of the dynamic slot algorithm based on the number of nodes is as follows:

1) The central node AP measures the number of nodes in the network in real time according to the actual situation in the network. When the number of nodes in the network changes, the AP broadcasts all nodes in the network and adjusts the corresponding network parameters (time slots, which are determined by Equation 5-(18) in Section 5.3.3 of this Chapter). When the number of nodes in the network has not changed, no broadcast is sent.

2) After receiving the broadcast sent by the AP, all the nodes adjust their time slots accordingly.

3) When a new node joins the network, it is first registered at the AP. The AP adjusts the network parameters, ie the length of the time slot, according to the number of nodes, and notifies all nodes in the form of broadcast, including the newly added nodes.

The difference from the existing algorithm is that the above work is only performed on the AP. The advantage of this is: First, since the AP does not have a hidden node problem, the AP can accurately listen to the information in the entire network. Therefore, the accuracy of the measurement can be improved; secondly, the overhead of adding measurement to the mobile node in the network is avoided. The scheme of using the AP to broadcast the adjusted parameters to all nodes in the network can effectively ensure that all nodes in the network use the same network parameters, and to some extent, ensure the fairness of the dynamic slot algorithm based on the number of nodes.

\section{Simulation analysis}

This simulation experiment uses the NS2 simulation tool to verify the performance of the proposed DSTBNN algorithm in a variety of network scenarios. This chapter focuses on throughput. In the network scenario proposed in this paper, each node only communicates with the central node (AP), and each node does not communicate with each other, but can detect each other's existence within the effective range. The routing protocol uses the DSDV protocol, and the propagation model uses the TwoRayGround ideal model with a simulation time of 50 seconds. If not specified, its parameters are set by the default parameters of NS-2.33.

This paper examines the impact of changes in the number of nodes in the network on network performance. The network scenario of the simulation results in all the graphs is as follows: firstly, the number of nodes in the network is kept unchanged, and then the number of nodes is increased to observe the influence on the system performance when the number of nodes increases. At the same time, the packet length is changed, and the comparison is performed under the packet lengths of 500 bytes, 1000 bytes, and 1500 bytes, respectively. IEEE 802.11 uses a fixed time slot of $20 \mu \mathrm{s}$.

As can be seen from Fig.1, when the node packet length is 1000 bytes, before the improvement (that is, using IEEE 802.11), when the number of nodes is at least 5, the total system throughput reaches $5.33 \mathrm{Mbit} / \mathrm{s}$; when the number of nodes is At 15:00, the system throughput was the smallest, 4.95 Mbit/s, a decrease of $0.38 \mathrm{Mbit} / \mathrm{s}$, a decrease of approximately $7.6 \%$. After using the dynamic slot algorithm based on the number of nodes, the total maximum throughput of the system is 5.43 Mbit/s when the number of nodes is 5, and the minimum throughput of the system is $5.02 \mathrm{Mbit} / \mathrm{s}$ when the number of nodes is 15 . In this case, the total throughput of the dynamic slot algorithm based on the number of nodes is higher than that of IEEE 802.11. 


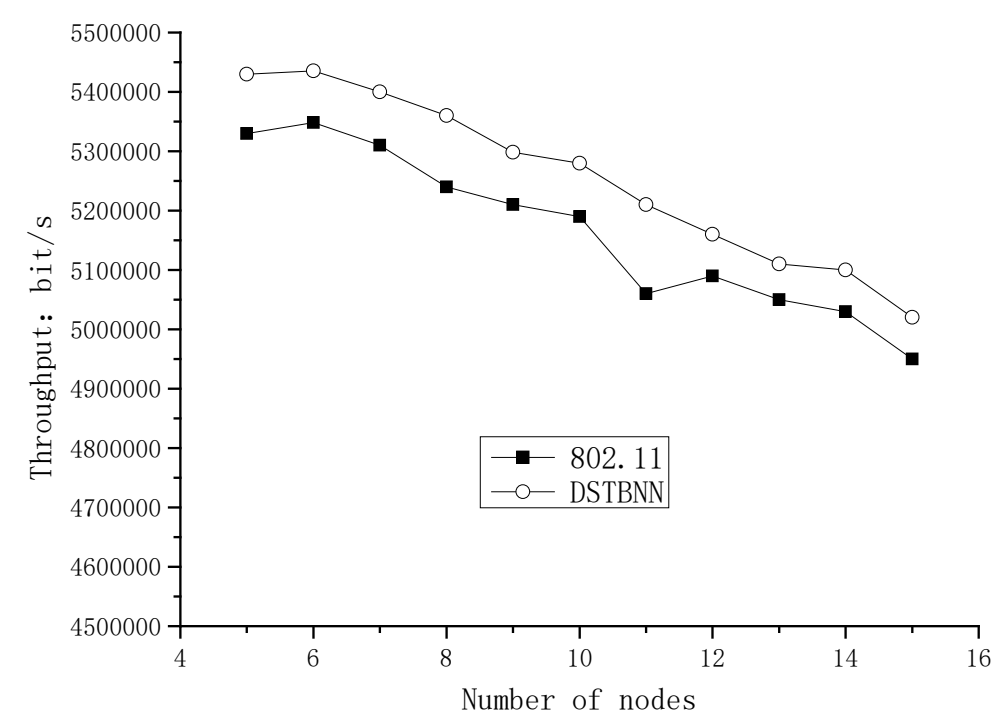

Fig.1 DSTBNN vs. 802.11

It can be concluded that when the packet length changes, when the number of nodes in the network increases, the total throughput of the IEEE 802.11 and the number of nodes based dynamic slot algorithm decreases, which is due to the increase in the number of nodes, resulting in increased collisions and effective channels. The utilization rate is reduced and the throughput is reduced. After using the dynamic slot algorithm based on the number of nodes designed in this paper, the total throughput is reduced, but it is larger than the IEEE 802.11 throughput. This is due to the dynamic slot algorithm based on the number of nodes. It can reduce the collision rate and correspondingly improve the effective utilization of the channel, thereby improving the total throughput and improving the network performance. This is consistent with the idea of dynamic slot algorithm based on the number of nodes designed in this paper. However, the dynamic slot algorithm based on the number of nodes is always larger than the total throughput of 802.11, and is relatively stable. The experiment further proves that in the case of multiple nodes, the dynamic slot algorithm based on the number of nodes can improve network performance.

\section{Conclusion}

In the IEEE 802.11 WLAN, as the number of nodes increases, the collision will increase accordingly, the channel utilization will decrease, and the total throughput will not increase and decrease, resulting in system performance degradation. In this paper, through an in-depth analysis of the root cause of the problem, this paper designs a simple and effective scheme-the dynamic time slot algorithm based on the number of nodes (DSTBNN). Finally, using NS2 simulation software, this paper has carried out several simulation experiments in various scenarios. The experimental results verify that the algorithm is simple, and the modification to IEEE 802.11 is small, which is suitable for use in wireless networks. Network system performance can be optimized based on changes in the number of nodes, improving system throughput and significantly improving network performance. According to the number and density of nodes, the algorithm dynamically adjusts the time slot, reduces the collision probability, and correspondingly improves the total network throughput, thus achieving the purpose of effectively improving network performance.

\section{Acknowledgements}

This research was supported by Doctor Initial Funding of Hubei University of Science and Technology (No. 2016-19XB003), NSFC-Big Data Science Center Project of Guangdong 
(U1611262), Research and Development Fund of Hubei University of Science and Technology (2018-19GZ01), the Scientific Research Project of Education Department of Hubei Province under Grant B2018179 and B2017181 and B2018175, the National Natural Science Foundation of China (No. 51479155).

\section{References}

[1] Xiao Y. A simple and effective priority scheme for IEEE 802.11. Communications Letters, IEEE, 2003, 7(2):70-72.

[2] Xiao Y. Backoff-Based priority schemes for IEEE 802.11. In: Proc. of the IEEE Int'l Conf. on Communications. Vol 3, 2003. 1568-1572.

[3] Xiao Y. Enhanced DCF of IEEE 802.11e to support QoS. Proc. of the IEEE Wireless Communications and Networking Conf 2003.1291-1296.

[4] Kim J H, Lee J K.Performance of carrier sense multiple access with collision avidance protocols in wireless lans.Wireless Personal Communications, 1999, 11(2):161-183.

[5] Bianchi G,Tinnirello I.Kalman filter estimation of the number of competing terminals in an IEEE 802.11 network[A]. INFOCOM'2003, 2003, 844-852.

[6] Bianchi G. Performance analysis of the IEEE 802.11 distributed coordination function. IEEE Journal on Selected Areas in Communications, 2000,18(3):535-547. 